\title{
Comparison of Methods for Counting Cells in Tissue Sections
}

\author{
John M. Basgen*, Silvia Rozen*, and Susanne Nicholas** \\ *Department of Pediatrics, University of Minnesota, Minneapolis, MN 55455 \\ **Department of Medicine, David Geffen School of Medicine at UCLA, Los Angeles, CA 90095
}

Researchers have long been interested in knowing the number of cells in normal and diseased organs. To be able to see and count the cells, the tissue often must be embedded in a medium such as paraffin or plastic, sectioned, and observed under a microscope. Because it is often not possible to differentiate the boundaries between cells even under the microscope, researchers have used nuclear counts as surrogates for cell counts. Organs and the nuclei they contain are 3-dimensional structures. When thin sections are made through the organ, one decreases the dimension of structures seen in the images of the sections. The 3-D organ and nuclei are not present, but 2-D profiles of the organ and nuclei are seen. The number of profiles of nuclei seen in the 2-D images is not directly related to the number of nuclei in the 3-D organ. Shape, volume, orientation, and number of nuclei determine the number of profiles seen in the 2-D image. Studies have tried to overcome this geometrical problem by relating the number of nuclear profiles to area of organ profile, or using modeling techniques, or shape factors $[1,2]$. These techniques have often resulted in conflicting data $[2,3]$. In 1917, Kittelson counted the number of glomeruli in rat kidneys. He exhaustively cut serial sections through the kidney and sequentially counted the glomeruli as they appeared. He thus obtained a direct count of all the glomeruli in the kidney independent of shape, size, and orientation of the glomeruli. This Exhaustive Count method is accurate but of course very time consuming.

In this study we compared the Exhaustive Count method to two other methods to try to find an accurate but faster counting method. We embedded a mouse kidney in PolyBed 812 (Polysciences, Inc.). We exhaustively cut $1-\mu \mathrm{m}$ sections from nine glomeruli using a Histo Jumbo diamond knife (Diatome US). An average of 79 sections were needed to completely cut through the glomeruli. Sections were stained with toluidine blue and digital images obtained using a Leitz Laborlux 8 microscope fitted with a 100X oil lens and a SPOT CCD camera. All sections through glomeruli were imaged. Adobe Photoshop software was used to observe the images. First, the Exhaustive Count method was used by sequentially observing all images through the glomeruli and counting the nuclei as they appeared (Fig. 1). An average of $213 \pm 22$ cells/glomerulus was counted. Next we used the Disector/Fractionator counting method [4]. This method uses a known fraction of the images through an organ, called the Sampling images. We used 1/5 of the images through each glomerulus. The image adjacent to each Sampling image is also used and known as the Look-up image. Profiles from nuclei seen in the Look-up section but not present in the Sampling section were counted and multiplied by the reciprocal of the sampling fraction to obtain the estimate of cell number (Fig. 2). An average of $211 \pm 29$ cells/glomerulus was counted using this method. A third method, the Weibel-Gomez counting method, uses the number of nuclear profiles per glomerular area, volume fraction of nuclei/glomerulus, and glomerular volume to estimate cell number [5]. Using this method an average of $235 \pm 26$ cells was counted. The Weibel-Gomez estimate was statistically different than the Exhaustive Count method $(\mathrm{p}=0.008)$. It overestimated the true cell count by an average $22 \pm 3(10 \%)$ cells/glomerulus and is thus a biased method. The Disector/Fractionator method was not statistically different from the Exhaustive Count method. It is thus an accurate and more efficient method for counting cells per glomerulus. 
[1] E. T. Bell, Am J Pathol. 19 (1936) 801.

[2] R. Østerby, Acta Medica Scand. (Suppl. 574) (1974) 1.

[3] N. Fukuhara, Tohoku J Exp Med. 95 (1968) 15.

[4] B. Pakkenberg, H. J. Gundersen, J Microsc. 150 (1988) 1.

[5] E.R. Weibel, Stereological Methods Vol. 1, Academic Press, London, 1979.

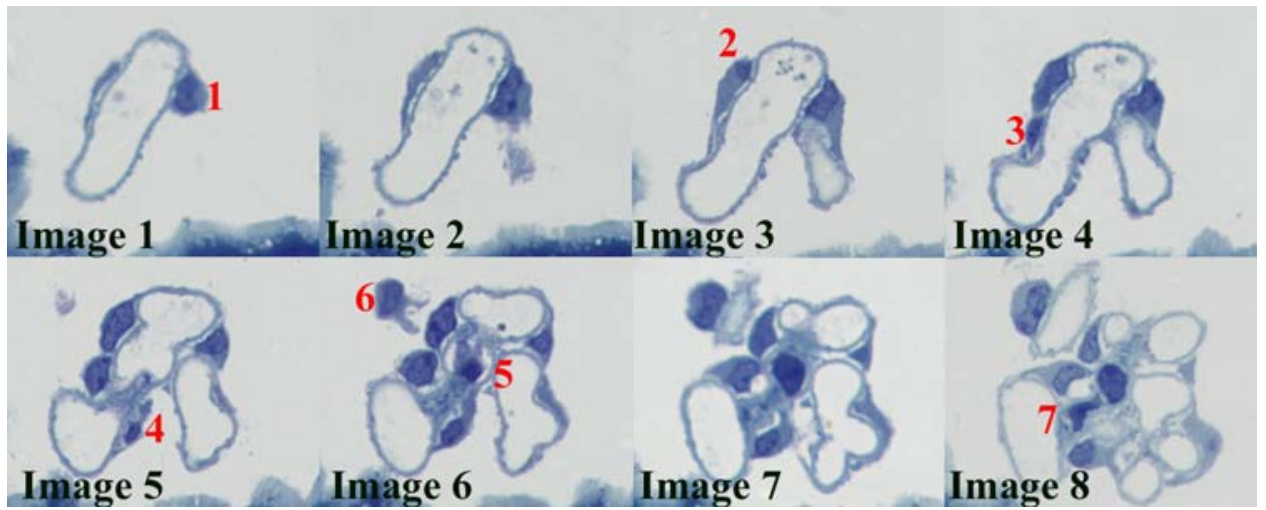

Fig. 1. First 8 images through a glomerulus showing the first 7 nuclei as counted with the Exhaustive Count method.

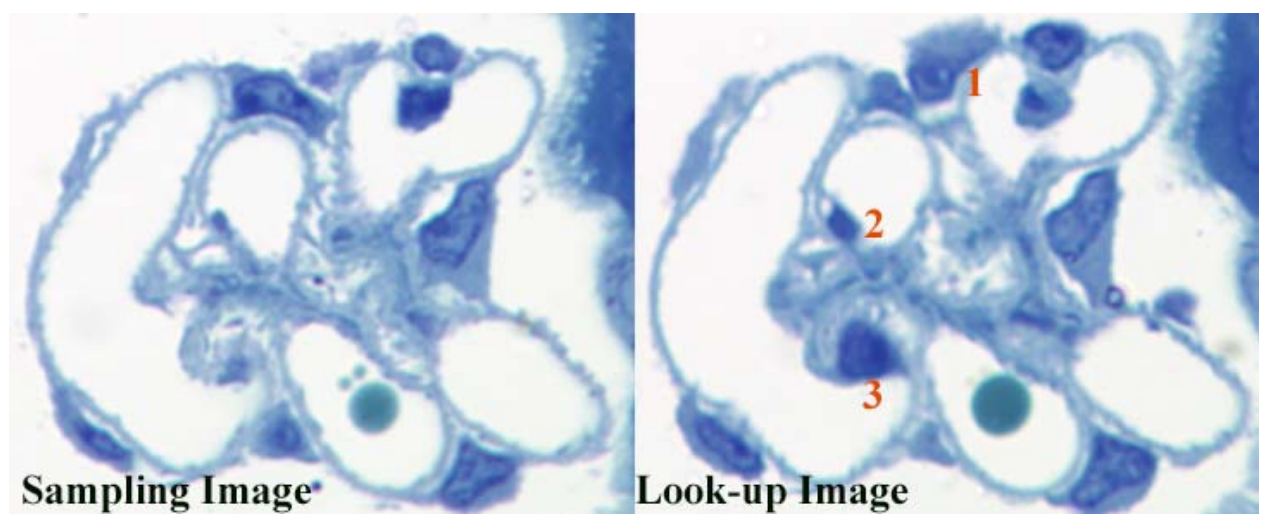

Fig. 2. Disector/Fractionator image pair where profiles from nuclei seen in the Look-up Image but not seen in the Sampling Image are counted.
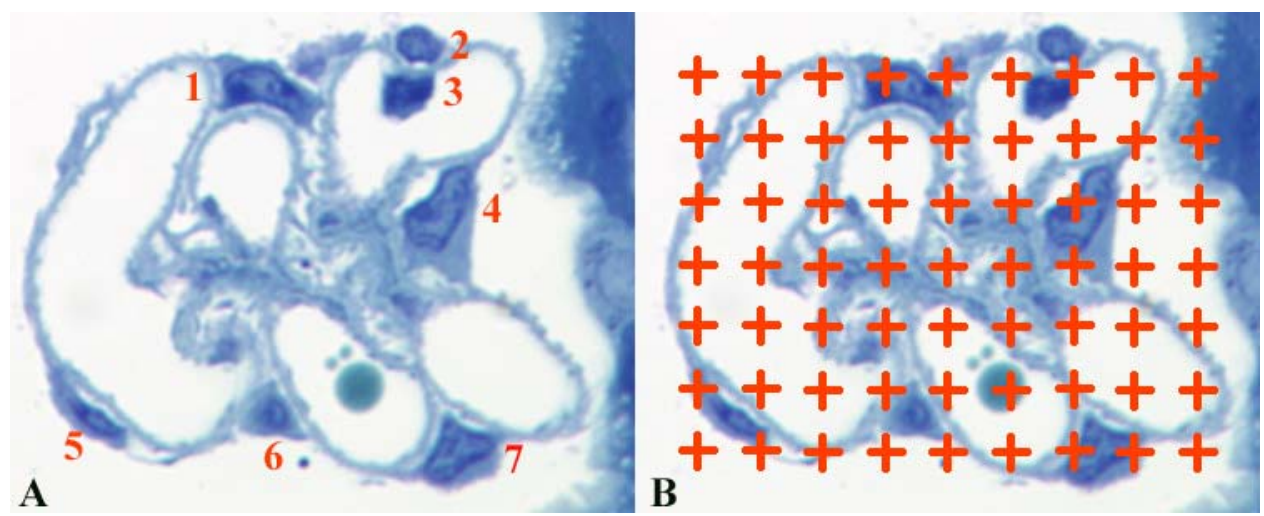

Fig. 3. The Weibel-Gomez method first counts the number of nuclear profiles (Panel A). Then the number of grid points hitting the glomerular profile and the number of points hitting the nuclear profiles are counted. 